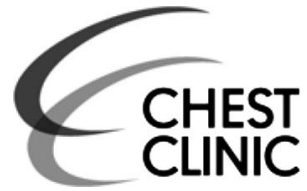

${ }^{1}$ Department of Medicine, Pulmonary and Critical Care Medicine, Albany Medical Center, Albany, New York, USA

${ }^{2}$ Department of Medicine, Pulmonary and Critical Care Medicine, Medical University of South Carolina, Charleston, South Carolina, USA

\section{Correspondence to} Dr Amit Chopra, Department of Medicine, MC-91, Albany Medical College, 47 New Scotland Avenue, Albany, NY 12208, USA; chopraa1@mail. amc.edu

Received 8 June 2016 Revised 22 August 2016 Accepted 5 September 2016 Published Online First 26 September 2016

\title{
The pressure-dependent air leak after partial lung resection
}

\author{
Amit Chopra, ${ }^{1}$ Peter Doelken, ${ }^{1}$ Marc A Judson, ${ }^{1}$ Terrill Huggins ${ }^{2}$
}

\section{CASE REPORT}

A 72-year-old man with a right middle lobe lung adenocarcinoma underwent lobectomy with removal of tube thoracostomy 3 days after the thoracic surgery. He presented approximately 3 weeks after surgery with increasing dyspnoea. A chest X-ray (CXR) revealed a moderately large, loculated basilar pneumothorax (figure 1A). A small bore tube thoracostomy was placed with reduction of the pneumothorax. However, a moderate-sized pneumothorax with a persistent air leak persisted for approximately 2 weeks after chest tube placement
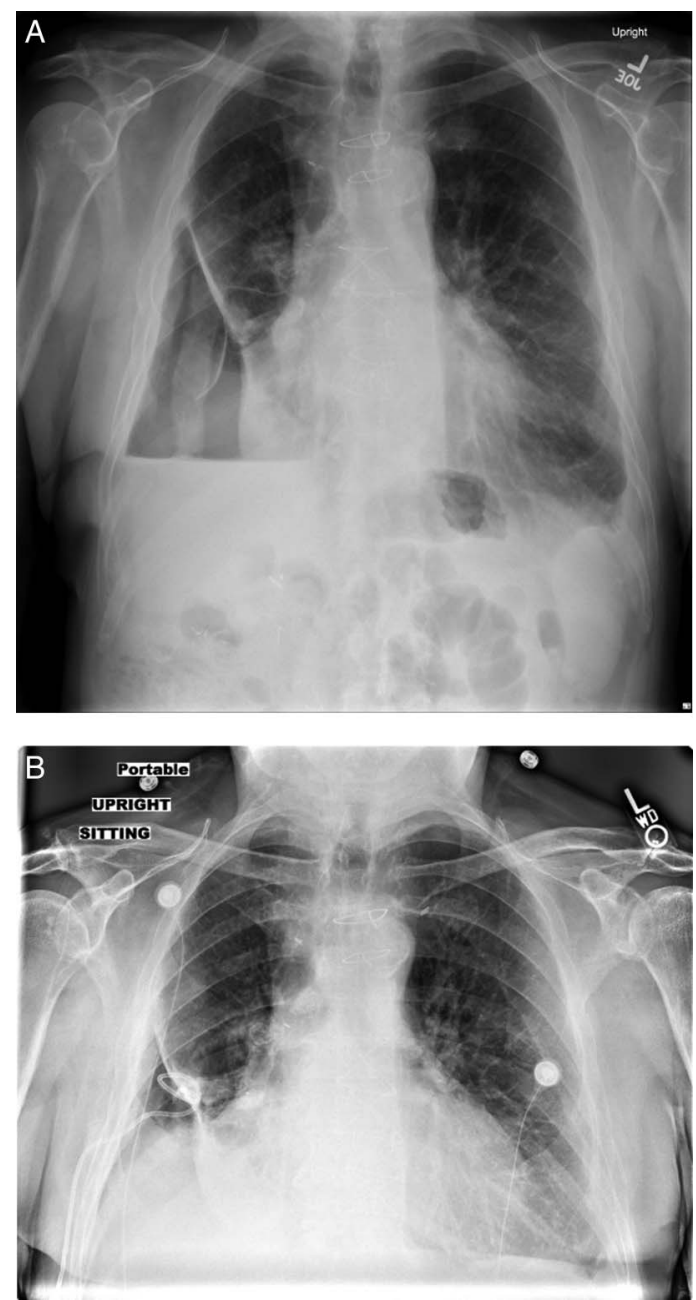

Figure 1 Chest X-ray. (A) Right basilar loculated pneumothorax 2 weeks after the right middle lobe resection. The patient developed a prolonged air leak. (B) Persistent loculated pneumothorax after chest tube placement with suction. The patient developed a prolonged small degree of air leak. requiring ongoing hospitalisation (figure 1B). Pleural manometry was performed sequentially, first with the chest tube open to water seal and then with chest tube clamped demonstrating that the air leak was pressure-dependent. Based on the manometric findings (figure 2), the chest tube was clamped and the pneumothorax remained radiographically stable. The tube thoracostomy was removed and the patient was discharged home. At 1-year follow-up, his CXR showed stable pneumothorax (figure 3).

\section{DISCUSSION}

Pneumothorax and air leak are common after partial lung resection. ${ }^{1}$ Prolonged air leak is associated with increased morbidity, hospital stay and increased health costs. $^{2-4}$ Pleural manometry allowed us to remove the chest tube early when an intermittent air leak was present. While the chest tube was patent, pleural manometry (figure 2) showed that the mean pleural pressure decreased after a cough due to discharge of air out of the pleural space. Subsequently, the pleural pressure rose and returned to baseline from air within the lung. This is a common and often an

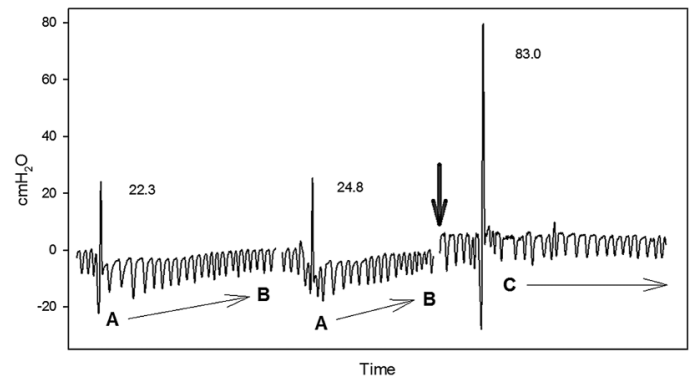

Figure 2 Pleural manometry tracing in a patient after post partial lung resection with prolonged air leak (PAL). $A$ baseline is obtained during quiet breathing with a chest tube open to water seal. The consecutive three spikes in pressure tracing represent a rise in pleural pressure due to cough. After cough, the pleural pressure immediately afterwards (A) is lower than baseline due to discharge of air through the open chest tube.

Subsequently, the pleural pressure rises to baseline (B) until the pressure gradient is ablated. However, if the chest tube is closed (marked by downward arrow) or in the absence of a chest tube, the air is not be able to be drained from the pleural space and, therefore, the pressure spike during cough is relatively higher and the pleural pressure post cough remains at baseline and stable; (C) this is because there is no pressure gradient. This manometry tracing proved that the air leak is pressure-dependent and that the chest tube could be safely removed. 


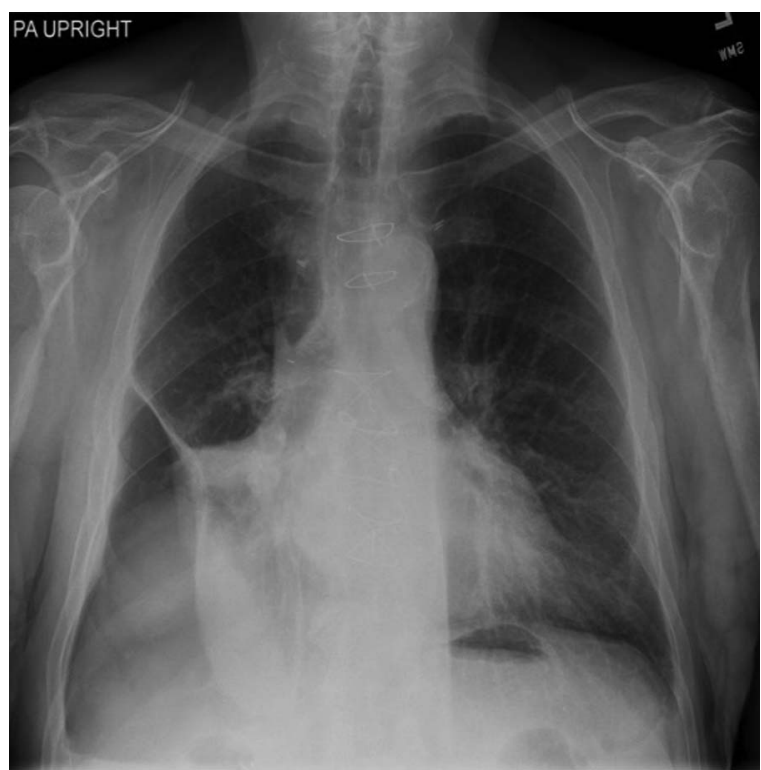

Figure 3 Follow-up chest X-ray (CXR). At approximately 1-year follow-up repeat CXR showed stable right basilar loculated pneumothorax.

underappreciated phenomenon. We suspect that the air originates in alveoli adjacent to the resected lung that are transiently over-distended by a reduction in pleural pressure. As this air enters the pleural space, the pressure gradient driving the air leak lessens and resolves, resulting in transient pressuredependent air leak (air leak resolves once the pressure gradient has abated). This phenomenon has been described in the pathophysiology of pneumothorax ex vacuo after thoracentesis in unexpandable lung. ${ }^{5}$ When the chest tube is clamped, the air cannot be drained from the pleural space and, other than a transient pressure spike during the cough, the pleural pressure remains unchanged and stable. Because the pleural pressure is unchanged, there is no decrease in pleural pressure to overdistended lung units to promote further air leak from the lung. These findings support a pressure-dependent pneumothorax, which does not result in progressively enlarging pneumothorax or tension pneumothorax and, therefore, does not need continued pleural drainage. In contrast, a pressure-independent air leak results in an enlarging pneumothorax with a continuous need for pleural drainage. We believe that this case illustrates a potential and novel use for pleural manometry to differentiate a pressure-dependent air leak from pressure-independent air leak, which is essential in determining if a chest tube can be safely removed. However, future prospective studies are required to establish the role of pleural manometry in the management of pneumothorax and air leak after partial lung resection. This manometric-based approach may allow a confident early removal of the chest tube and reduce hospital length of stay after surgery.

Contributors All co-authors have contributed in drafting of this manuscript.

Competing interests MAJ: consultant for Janssen, Celgene, Questcor, Mistubishi-Tanabe, Novartis.

TH: Grant for IPF trial from InterMune, Roche and Boerhinger Ingelheim. No direct $\mathrm{COI}$ related to manuscript.

\section{Patient consent Obtained.}

Provenance and peer review Not commissioned; externally peer reviewed.

\section{REFERENCES}

1 Lois M, Noppen M. Bronchopleural fistulas: an overview of the problem with special focus on endoscopic management. Chest 2005;128:3955-65.

2 Coughlin SM, Emmerton-Coughlin HM, Malthaner R. Management of chest tubes after pulmonary resection: a systematic review and meta-analysis. Can I Surg 2012;55:264-70

3 Cerfolio RJ, Bryant AS. The management of chest tubes after pulmonary resection. Thorac Surg Clin 2010;20:399-405.

4 Cerfolio RJ, Bryant AS, Singh S, et al. The management of chest tubes in patients with a pneumothorax and an air leak after pulmonary resection. Chest 2005;128:816-20.

5 Heidecker J, Huggins JT, Sahn SA, et al. Pathophysiology of pneumothorax following ultrasound-guided thoracentesis. Chest 2006;130:1173-84. 